\title{
GLICOSÍMETRO DIGITAL APLICADO A ANÁLISE DE AÇÚCAR REDUTOR EM POLPA DE FRUTAS
}

\section{DIGITAL GLUCOMETER APPLIED TO THE ANALYSIS OF REDUCING SUGAR IN FRUIT PULP}

\author{
Tércio da Silva de Souza ${ }^{1}$ \\ Jéssika Santos de Oliveira ${ }^{1}$ \\ Luiz Claudio Cindra de Souza ${ }^{2}$

\begin{abstract}
${ }^{1 *}$ Instituto Federal de Educação, Ciência e Tecnologia do Espírito Santo - Campus de Alegre, Brasil. E-mail: tssouza@ifes.edu.br

${ }^{2}$ Licenciada em Ciências Biológicas, Instituto Federal de Educação, Ciência e Tecnologia do Espírito Santo Campus de Alegre, Brasil. E-mail: jessikaoliveira664@gmail.com

${ }^{3}$ Graduando em Oceanografia, Universidade Federal do Espírito Santo - Campus de Vitória, Brasil. E-mail: luizccindras@gmail.com * autor para correspondência
\end{abstract}

Artigo submetido em 05/08/2020, aceito em 18/12/2020 e publicado em 19/04/2021.

Resumo: A análise de açúcares em alimentos é de suma importância no controle da qualidade dos produtos, sendo estes responsáveis pelo flavor, cor e textura. Em polpas de frutas a doçura é resultante da proporção entre a frutose, glicose e a sacarose, estes possuem poder adoçante diferenciado e definem o sabor final do produto. Existe uma ampla lista de técnicas e métodos oficiais capazes de determinar a concentração de açúcares em amostras de alimentos, contudo em sua maioria são de custo levado, geram grande volume de resíduos e seus resultados são demorados. Neste trabalho, propôs-se desenvolvimento uma metodologia para quantificação de açúcares redutores (AR) em amostras de polpa de frutas com glicosímetro digital "On Call Plus ${ }^{\circledR}$. A adaptação e validação da metodologia consistiram na construção de curva de calibração com padrões de glicose e verificação do efeito da interferência de outros açúcares na quantificação do teor de glicose; análise de amostras de polpa de fruta com glicosímetro digital e comparação com método de referência ADNS (Ácido 3,5dinitrosalicílico), e por fim, o tratamento dos dados e avaliação dos parâmetros de validação para metodologia. O uso do glicosímetro digital foi validado, revelando-se rápido, seguro, de baixo custo, relativamente preciso para a quantificação de açúcar redutor em amostras de polpa de fruta.

Palavras-chave: polpa de frutas; glicosímetro digital; açúcar solúvel redutor

Abstract: An analysis of sugar in foods is an important paramount in the quality control of products, which are responsible for the flavor, color and texture. In fruit pulps, sweetness results from the proportion between fructose, glucose and sucrose. These have differentiated sweetening power and define the final flavor of the product. There is an extensive list of official techniques and methods capable of determining the concentration of sugar in the samples, but most are expensive, generate a large volume of waste and their results take time. This work aims to develop a methodology for quantifying reducing sugars (AR) in fruit pulp sample with a digital glucometer "On Call Plus®". An adaptation and validation of the methodology consisted in the construction of a calibration curve with a glucose standard and verification of the effect of the interference of other sugars in the quantification of the glucose content; analysis of fruit pulp samples with digital glucometer and comparison with the 
ADNS reference method (3,5-dinitrosalicylic acid) and, finally, data treatment and evaluation of the methodology validation. The use of the digital glucometer has been validated, proving to be fast, safe, low cost, relatively accurate for the quantification of reducing sugars in fruit pulp sample.

Keywords: fruit pulp; digital glucometer; reducing soluble sugar.

\section{INTRODUÇÃO}

Os principais açúcares presentes nas frutas são a frutose, a glicose e a sacarose. Estes diferem significantemente em doçura, e juntamente com ácidos orgânicos presentes atuam influenciando diretamente no sabor e aroma da fruta e dos derivados (BAIQUAN et al., 2015).

SILVA et al. (2003) diferem em categoria distintas as espécies de açucares, sendo: os monossacarídeos, glicose e frutose são açúcares redutores, pois possuem grupo carbonílico e cetônico livres (carbono anomérico) capazes de se oxidarem na presença de agentes oxidantes em soluções alcalinas (Figura 1); e os dissacarídeos, caso da sacarose, que não possuem essa característica sem sofrerem hidrólise da ligação glicosídica são denominados de açúcares não redutores.

Figura 1: Monossacarídeos frutose e glicose em estrutura de ciclizada com indicação do carbono anomérico.
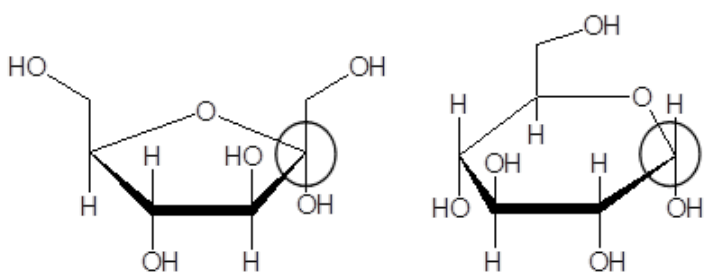

Fonte: Autor (2020)

A análise de açúcares em alimentos é de suma importância no controle da qualidade dos produtos, além dos mesmos serem responsáveis pelo flavor, pela cor e pela textura do produto. O conhecimento da composição qualitativa e quantitativa dos açúcares em um alimento auxilia no controle de qualidade e no atendimento das características impostas pela legislação para o produto (KOKKINIDOU et al., 2018; CURI et al. 2017; SENGAR e SHARMA, 2014).

Desta forma, a determinação do tipo e concentração dos açúcares nos alimentos é importante, visando fornecer as informações nutricionais e calóricas que são requeridas impressas nas embalagens; para o controle de qualidade de forma que assegure o cumprimento da legislação, identificando possíveis produtos adulterados (CALDAS et al., 2015 e ZHANG, et al., 2015).

Atualmente existe uma ampla lista de técnicas e métodos oficiais capazes de determinar a concentração de açúcares redutores e açúcares totais em amostras. Os métodos mais utilizados para a determinação de açúcares podem ser agrupados em refratométrico ( ${ }^{\circ}$ Brix), titulométricos (EDTA, Lane-Enyon e LuffSchoorl), gravimétricos (Musson-Walker) e espectrofotométricos (ADNS-Ácido 3,5dinitrossalicílico, Antrona, Fenol-Sulfúrico, Somogyi-Nelson) e os métodos cromatográficos (Cromatografia em Papel, Cromatografia Gasosa e CLAECromatografia Líquida), sendo este último, o mais utilizado (DORNEMANN, 2016; SILVA et al., 2003).

Os métodos acima citados, com exceção do refratômetro, são de custo elevado, e geram grande volume de resíduos e seus resultados são demorados, o que passa a ser um problema para a indústria de processamento de alimentos, em especial no controle da qualidade de produção. Esta etapa requer a tomadas de decisões rápidas o que os métodos usuais não oferecem (SOUSA et al., 2020; BRASIL et al., 2016).

Quando se deseja analisar grande número de amostras por unidade de tempo, melhorar a exatidão dos resultados 
analíticos com menor tempo, reduzir a quantidade de amostras e reagentes, eliminar algumas possibilidades de contaminação, diminuir o custo operacional e empregar método de análise específico, deve-se considerar a adaptação e/ou substituição dos métodos tradicionais (GONZÁLEZ e HERRADOR, 2007; DEMIATE et al., 2002) através da busca por novas tecnologias.

Dessa forma, visando desenvolver uma metodologia para quantificação de açúcares solúveis que possibilite a realização de análises em larga escala, com equipamento de fácil aquisição, rapidez e custo relativamente baixo, este trabalho propõe a utilização de glicosímetro digital para quantificação de açúcares redutores (AR) em amostras de polpa de fruta.

\section{PROCESSOS METODOLÓGICOS}

O glicosímetro utilizado nesse estudo foi o On Call Plus ${ }^{\circledR}$. O aparelho realiza a leitura na faixa de medição de 20 a $600 \mathrm{mg} \mathrm{dL}^{-1}$ e possui a opção para armazenar os dados no computador. A tela do medidor é pré-ajustada para concentrações de glicose em mmol $\mathrm{L}^{-1}$ ou $m g \mathrm{dL}^{-1}$ e o resultado é fornecido em 10 segundos, custa no mercado nacional de $\mathrm{R} \$$ 49,90 e 61,40 e a caixa com 100 unidades de tiras de medição em torno de R $\$ 120,00$.

Esse aparelho é indicado para mensurar sangue capilar e utiliza o sistema amperométrico glicose-oxidase dependente, com necessidade de $u m \mu \mathrm{L}$ (um microlitro) de amostra para avaliação.

No sistema de medição amperométrico, a tira-teste reagente inserida no aparelho quando em contato com a amostra de sangue (glicose) sofre reação eletroquímica, em que a intensidade da corrente elétrica resultante é proporcional à concentração de glicose na amostra (OLIVEIRA et al., 2015).

As tiras para teste são finas e possuem um sistema eletroquímico com dois eletrodos de trabalho e um de referência. Depois que a tira é inserida no medidor, aplica-se a amostra na parte final da tira, esta chega aos eletrodos por difusão. O eletrodo de trabalho é recoberto com a enzima glicose oxidase, que na presença de glicose gera uma reação redox com geração de corrente elétrica. A corrente elétrica gerada na reação é proporcional à concentração de glicose presente na amostra, esta é calculada com base em uma solução padrão de glicose (HARRIS, 2010).

A adaptação e validação do uso do glicosímetro para quantificação de açúcares redutores (AR) em amostras de frutas na forma de polpa foi realizada em quatro etapas: Construção de curva de calibração com concentrações conhecidas de glicose; Verificação do efeito de interferentes na quantificação do teor de glicose em solução padrão; Quantificação dos teores de açucares redutores em amostras de polpa de frutas utilizando o método do glicosímetro e o método ADNS; Tratamento dos dados e a avaliação dos parâmetros de validação.

\subsection{CONSTRUÇÃO DA CURVA DE CALIBRAÇÃO DE AÇÚCARES REDUTORES COM PADRÃO DE GLICOSE}

A curva de calibração para análise de açúcares redutores foi construída a partir de soluções padrão de glicose, preparadas em tampão fosfato $\left(0,1 \mathrm{~mol} \mathrm{~L}^{-1}, \mathrm{pH}=7,2\right)$, com concentrações variando de 20-600 mg $\mathrm{dL}^{-1}$. A análise dos padrões foi realizada pelo método do glicosímetro e também pelo método do ADNS (VASCONCELOS et al., 2013 e SANTOS et al., 2017). Os dados obtidos foram submetidos à análise descritiva e de regressão, utilizando o Programa R, versão 3.0.2 (Team R. C. 2013).

A sensibilidade (SENS) da curva de calibração empregada neste trabalho foi medida pelo coeficiente angular da equação de regressão da calibração e a linearidade (LIN), pelo coeficiente de correlação. Os 
limites de detecção (LOD - Equação 1) e os limites de quantificação (LOQ - Equação 2) dos métodos foram calculados por meio das duas equações subsequentes (GONÇALVES et al., 2010; GONZÁLEZ e HERRADOR, 2007, IMOTO et al., 2004; BRITO et al., 2003).

\section{$\mathrm{LOD}=\left(\left(3 * S^{2}\right)\right) /$ SENS \\ $\mathrm{LOQ}=\left(\left(10 * \mathrm{~S}^{2}\right)\right) / \mathrm{SENS}$}

Equação 1

Equação 2

Onde: $S^{2}$ corresponde ao desvio padrão das concentrações do analito para a curva de regressão base do método e o SENS corresponde à sua sensibilidade.

\subsection{EFEITO DA INTERFERÊNCIA DE OUTROS AÇÚCARES NA QUANTIFICAÇÃO DE GLICOSE}

Para verificar a interferência de outros açúcares presentes em amostras, na quantificação da glicose pelo método do glicosímetro foi analisada além de solução padrão de glicose, a mistura desta, com soluções padrão de frutose e/ou sacarose na proporção $\left(1: 1 \mathrm{v} \mathrm{v}^{-1}\right)$, conforme descrito na Tabela 1.

Os resultados obtidos foram submetidos a teste de normalidade de Shapiro-Wilk, homocedasticidade de Bartlett e análise de variância (ANOVA) utilizando Programa R, versão 3.0.2 (Team R. C. 2013).

\subsection{QUANTIFICAÇÃO DO TEOR DE AÇUCARES REDUTORES EM AMOSTRAS DE POLPA DE FRUTA UTILIZANDO O MÉTODO DO GLICOSÍMETRO E O MÉTODO ADNS.}

Amostras de polpa de fruta comerciais (amora, banana, goiaba, graviola, jabuticaba, maçã, manga, mamão, maracujá, melão, morango, pera e laranja) foram adquiridas no mercado local congeladas em embalagem de 100g, em seguida foram descongeladas e submetidas à quantificação de açúcares redutores.

Para realizar a extração do AR, um grama de amostra foi dissolvida em $100 \mathrm{~mL}$ (um dL) de solução tampão fosfato $0,1 \mathrm{~mol}$ $\mathrm{L}^{-1}, \mathrm{pH}=7,2$ e deixada em repouso por 10 minutos. Decorrido esse tempo foi realizado filtração a pressão reduzida utilizando papel de filtro quantitativo.

A quantificação pelo método do glicosímetro foi feita através da leitura direta das amostras em glicosímetro conforme orientação do fabricante. Procedimento idêntico ao utilizado na mensuração de glicose no sangue, introduzindo a fita medidora na solução filtrada e tamponada da polpa de fruta.

Já no método ADNS (VASCONCELOS et al., 2013 e SANTOS et al., 2017), $400 \mu \mathrm{L}$ de cada amostra foi incubada juntamente com $400 \mu \mathrm{L}$ de reagente ADNS em banho-maria por 5 minutos a $100^{\circ} \mathrm{C}$. Resfriou-se a mistura em banho de gelo, adicionou-se 7,2 mL de água destilada e efetuou-se a leitura das absorbâncias a 540 nm em espectrofotômetro AGILENT CARY60 UV-VIS.

As medidas foram realizadas em triplicata e a concentração de AR em ambos os métodos foram calculadas com base nas respectivas curvas de calibração com padrões de glicose.

\subsection{TRATAMENTO DOS DADOS E A AVALIAÇÃO DOS PARÂMETROS DE VALIDAÇÃO PARA METODOLOGIA.}

Os valores de AR obtidos pelos dois métodos foram submetidos à análise descritiva e análise de correlação Pearson. Para gerar os resultados esperados, foi efetuada análise de regressão e correlação utilizando o Programa $\mathrm{R}$, versão 3.0.2 (Team R. C. 2013). 


\section{REVISTA}

Tabela 1. Avaliação do efeito de interferentes na quantificação de glicose pelo método do glicosímetro.

\begin{tabular}{llll}
\hline \multirow{2}{*}{ Interferentes } & Glicose & Frutose & Sacarose \\
\cline { 2 - 4 } & \multicolumn{3}{c}{ Concentração $\left(\mathrm{mg} \mathrm{dL}^{-1}\right)$} \\
\hline Ensaio 1 & 300 & - & - \\
& 300 & - & - \\
& 300 & - & - \\
\hline Ensaio 2 & 300 & - & 200 \\
& 300 & - & 300 \\
& 300 & - & 400 \\
\hline Ensaio 3 & 300 & 200 & 200 \\
& 300 & 300 & 300 \\
& 300 & 400 & 400 \\
\hline Ensaio 4 & 300 & 200 & - \\
& 300 & 300 & - \\
& 300 & 400 & - \\
\hline
\end{tabular}

Fonte: Autor (2020).

Fonte: Autor (2020).

\section{RESULTADOS E DISCUSSÃO}

Inicialmente a curva de calibração foi construída com os dados obtidos na leitura dos padrões glicose na faixa 20 a 600 mg $\mathrm{dL}^{-1}$, que é a faixa indicada pelo fabricante do glicosímetro (Figura 2), porém os pontos extremos, abaixo de $100 \mathrm{mg} \mathrm{dL}^{-1}$ e acima de $500 \mathrm{mg} \mathrm{dL}^{-1}$ não se encontram dentro do comportamento esperado, houve um desvio de linearidade. Logo, não seria ideal trabalhar nessas faixas de concentração, pois acarretará um maior erro associado.

Para uma melhor resposta e linearidade da curva os pontos extremos foram retirados, pois de acordo com González e Herranor et al. (2007), em métodos quantitativos de análise os primeiros valores da faixa podem ser os valores dos limites de detecção e de quantificação e os últimos dependem do sistema de resposta de medição, o que pode não corresponder a faixa ótima do equipamento.

Utilizando a faixa de 100 a $550 \mathrm{mg}$ $\mathrm{dL}^{-1}$ os dados quando submetidos aos testes de normalidade e homocedasticidade atenderam as suposições básicas da ANOVA (análise de variância) para a regressão linear na construção da curva de calibração do glicosímetro (Figura 3), por isso esta foi a faixa utilizada.

Os resultados obtidos na ANOVA para regressão da curva de calibração ajustada (Figura 3) indicaram que esta é significativa pelo teste $\mathrm{F}$, com $p$ value $<2,2$ $10^{-16}$. A curva (Equação 3) explica significativamente $\quad 99,39 \%$ do comportamento observado para os pontos, podendo então ser usada para estimar o teor de AR através da mensuração com o glicosímetro.AR (mg $\left.\mathbf{d L}^{-1}\right) \quad=$ \{[(LEITURA)+152,89]/0,6358\} Equação 3

A curva de calibração para método de quantificação de AR através do 
glicosímetro foi validada pela análise da sua sensibilidade (SENS), da sua linearidade (LIN) e dos seus limites de detecção (LOD) e quantificação (LOQ) em comparação ao método ADNS (Tabela 2).

A validação da curva de calibração consiste em demonstrar que o método analítico foi adequado para o seu propósito, considerando as adaptações (inclusão de novas técnicas ou uso de diferentes equipamentos), quando comparando com metodologias já validadas (GONZÁLEZ e HERRADOR, 2007; IMOTO et al., 2004; BRITO et al., 2003).

Segundo Figueiredo (2012), a linearidade é a capacidade de uma metodologia analítica demonstrar que os resultados obtidos são diretamente proporcionais à concentração do analito na amostra, dentro de um intervalo específico e, a sensibilidade é um parâmetro que demonstra a variação da resposta em função da concentração do analito.

González e Herranor (2007) define LOQ como a mais baixa concentração de analito que pode ser determinada quantitativamente com um nível aceitável de precisão e exatidão e o LOD, a menor concentração do analito em uma amostra que pode ser detectada, mas não necessariamente quantificada sob as condições estabelecidas do teste.

Os resultados dos parâmetros de validação indicaram forte linearidade para o método avaliado. Quanto à sensibilidade este método apresentou menor sensibilidade, e com limites de detecção mais elevados que o método ADNS, contudo esta limitação é compensada pela praticidade, economia de material de laboratório e menor geração de resíduo para os fins desejados.

Tabela 2. Parâmetros de validação parcial da curva de calibração calculados para o método do glicosímetro e para o método referência ADNS.

\begin{tabular}{|c|c|c|c|c|}
\hline \multirow[b]{2}{*}{ Método } & \multirow[b]{2}{*}{ SENS } & \multirow[b]{2}{*}{ LIN } & \multicolumn{2}{|c|}{ LOD LOQ } \\
\hline & & & & $\left.\mathrm{dL}^{-1}\right)$ \\
\hline Glicosímetro & 0,6358 & 0,9939 & 26 & 122 \\
\hline ADNS & 0.3726 & 0,9964 & 10 & 35 \\
\hline
\end{tabular}

Fonte: Autor(2020).

Em relação a especificidade, esta é definida pela capacidade do método em detectar o analito de interesse na presença de outros componentes da matriz (GONÇALVES JUNIOR, 2001). Para demonstrar a especificidade do método, a análise pode ser realizada seguida por outras técnicas comprobatórias ou utilizando método de adição de padrão analítico ou comparação com padrão externo (FIGUEREDO, 2012).

Para verificar o efeito de interferentes na mensuração de AR com o glicosímetro foi utilizado a técnica da adição de padrão analítico (frutose e sacarose) à solução de glicose e verificado a interferência destes nos resultados. Os teores mensurados (Figura 3) foram submetidos aos testes de normalidade e homocedasticidade e atenderam as suposições básicas para utilização de teste paramétrico.

Quando avaliados os efeitos do tipo de interferente e sua concentração, pode-se constatar que as médias não apresentaram diferenças significativas pelo teste $F$ (ANOVA), $p$-value $=1,657$. Sendo assim, a espécie interferente e a sua concentração não afetam diretamente a medição de $\mathrm{AR}$ usando o glicosímetro. Este fato pode ser explicado pela especificidade da enzima glicose oxidase contida nas tiras analisadoras pela glicose.

A presença dos interferentes provocou um aumento sistemático, em média, da leitura com e sem interferentes (Figura 4). A análise de variância apresentou diferença entre médias significativas pelo teste $\mathrm{F}$, com $p$-value = 0,739 e o teste de comparação de Dunnett 
indicou diferença entre médias com interferentes e o controle contendo somente glicose.
A leitura da concentração de AR com glicosímetro apresentou um incremento médio de 40,7 $\mathrm{mg} \mathrm{dL}^{-1}$.

Figura 2. Curva de calibração para análise de açúcar redutor com glicosímetro digital.

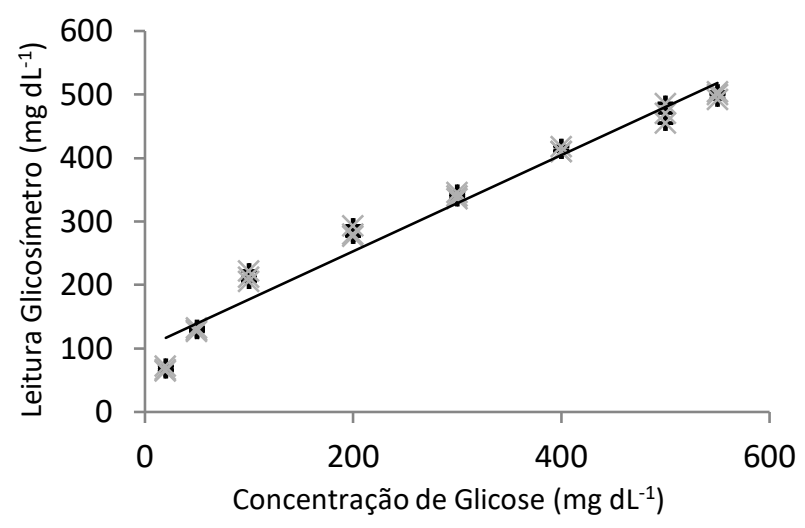

Fonte: Autor (2020).

Figura 3. Curva de calibração ajustada para análise de açúcar redutor com glicosímetro digital.

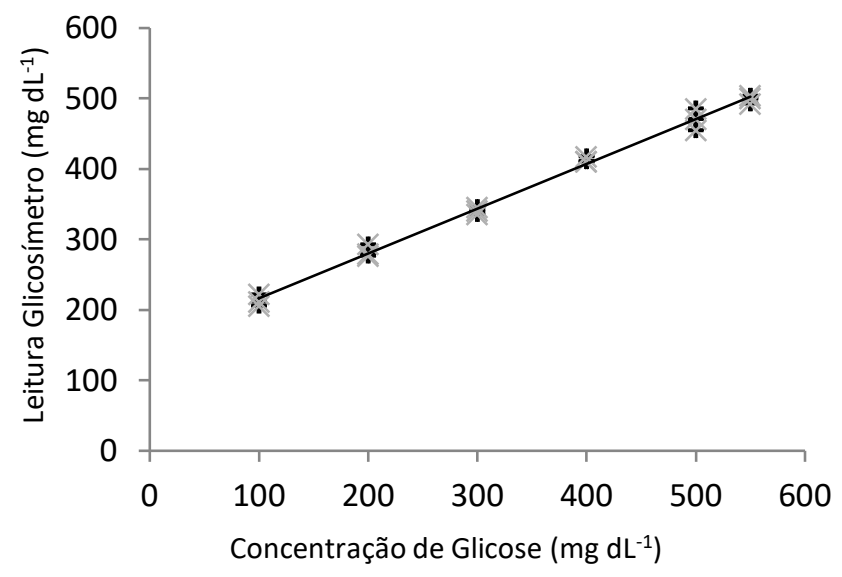

Fonte: Autor (2020).

De posse destas informações foi necessário construir uma nova equação que permite ajustar a equação 3 para a leitura de AR realizada no glicosímetro para estimar o teor de AR em amostras de polpa de fruta (Equação 4).

$\operatorname{AR}\left(\mathbf{m g ~ d L}^{-1}\right)=$

$\{[(($ LEITURA)+152,89)]/0,6358]-40,7\}

\section{Equação 4}

Para validação da equação de ajuste foi preciso avaliar o grau de correlação entre os teores de AR presente nos padrões obtidos pelo método do glicosímetro e pelo método do ADNS e assim inferir na precisão que o modelo proposto se relaciona ao método de referência (GUIMARÃES, 2017). 
Figura 3: Teor de açúcar redutor (AR) medido com glicosímetro em solução de glicose na presença de interferentes.
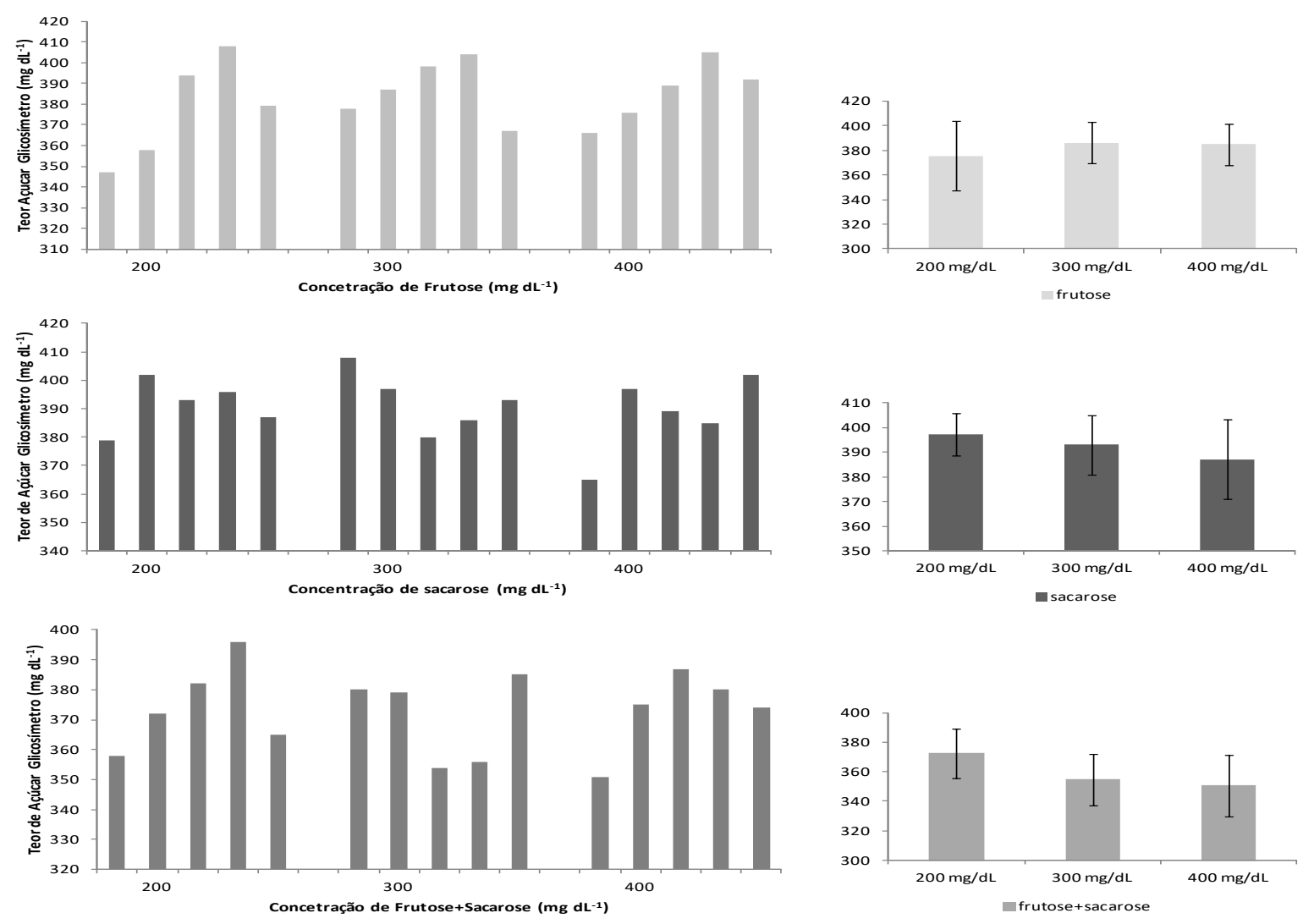

Fonte: Autor (2020).

Figura 4: Teor de açúcar redutor (AR) medido com glicosímetro em solução de glicose na presença e ausência de interferentes.

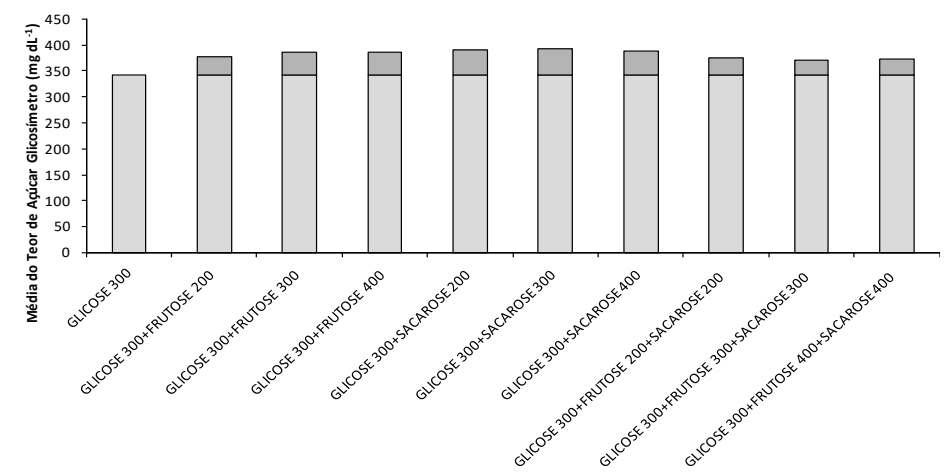

Fonte: Autor (2020).

Figura 5: Comparação entre a concentração de AR em diferentes amostras pelo método do glicosímetro e ADNS. 


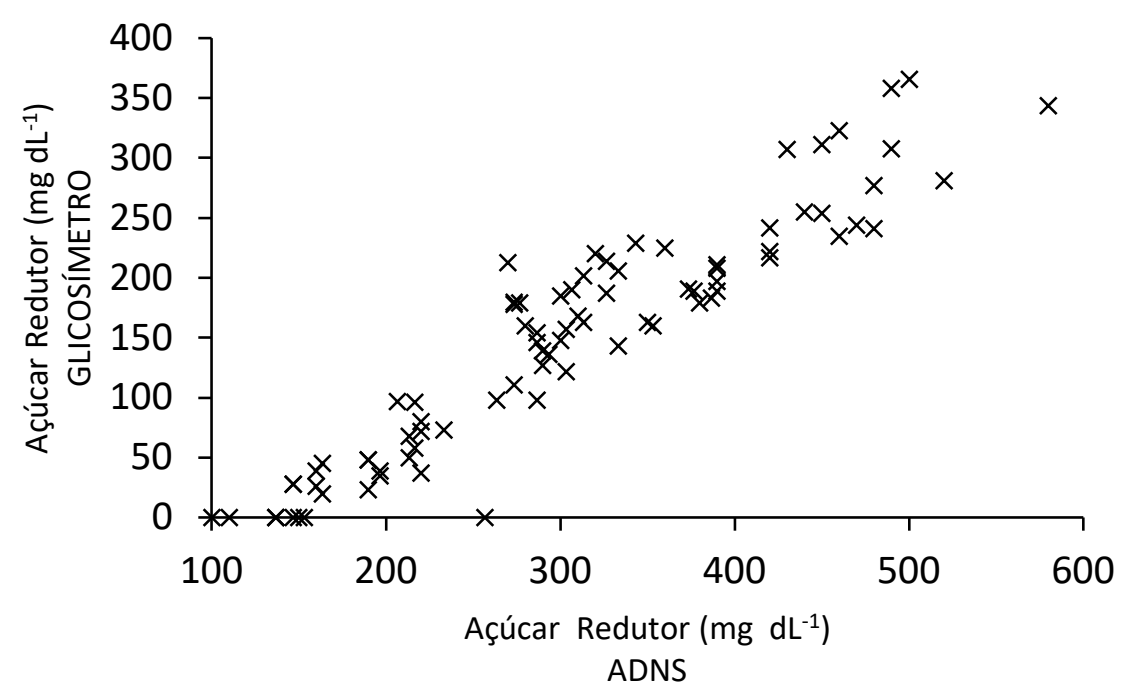

Fonte: Autor (2020).

Os valores de AR obtidos pelos dois métodos foram submetidos à análise de correlação de Pearson. O coeficiente de correlação $(\hat{r}=0,95)$ indicou que as variáveis estão positivamente relacionadas e a variância compartilhada indica uma forte correlação linear (Figura 5). A elevada correlação entre os dois métodos permitiu o ajuste dos valores medidos de AR pelo glicosímetro nas amostras de polpa de fruta através da equação 4.

Dessa forma, o glicosímetro On Call Plus ${ }^{\circledR}$ utilizado nesse trabalho se mostrou funcional para estimar teor de AR em amostras de polpa de frutas com alto grau de praticidade e confiabilidade. Com quantidade de amostra requerida para análise pequena, capacidade operacional variando de 20 e 30 amostras por hora, com custo de três a cinco reias por amostras e pequeno quantitativo de resíduo, sendo este de fácil manuseio e tratamento.

\section{CONCLUSÕES}

O método de uso do glicosímetro digital On Call Plus ${ }^{\circledR}$ foi validado, revelando-se rápido (20-30 amostra $\mathrm{h}^{-1}$ ), seguro (pequena quantidade de resíduo e de baixa periculosidade), de baixo custo (R\$ 3,00-5,00 amostra $\left.^{-1}\right)$, relativamente preciso e bastante eficiente para a quantificação de AR em polpa de frutas. A equação de ajuste $\mathrm{AR} \quad\left(\mathrm{mg} \quad \mathrm{dL}^{-1}\right)$ = $\{[(($ LEITURA $)+152,89)] / 0,6358]-40,7\}$ mostrou-se com alto grau confiabilidade para e especificidade, com SENS $=0,6358$; $\mathrm{LIN}=0,9939 ; \mathrm{LOD}=26\left(\mathrm{mg} \mathrm{dL}^{-1}\right), \mathrm{LOQ}=$ $122\left(\mathrm{mg} \mathrm{dL}^{-1}\right)$.

\section{AGRADECIMENTOS}

Agradecer ao Instituto Federal do Espírito Santo - IFES, a Universidade Federal do Espírito Santo - UFES, a Fundação de Amparo ao Pesquisador Espírito Santo (FAPES), à Coordenação de Aperfeiçoamento de Pessoal do Nível Superior (CAPES) e ao Conselho Nacional de Desenvolvimento Científico e Tecnológico (CNPQ) pelo apoio logístico e financeiro.

\section{REFERÊNCIAS}

BAIQUAN, Ma. et al. Comparative assessment of sugar and malic acid composition in cultivated and wild apples.

Food chemistry, v. 172, p. 86-91, 2015. https://doi.org/10.1016/j.foodchem.2014.09. $\underline{032}$. 
BRASIL, Alexandre Silva et al. Evaluation of physicochemical quality of frozen fruit pulp marketed in the city of Cuiabá-MT.

Revista Brasileira de Fruticultura, v. 38, n. 1, p. 167-175, 2016

https://doi.org/10.1590/0100-2945-253/14.

BRITO, Natilene Mesquita et al. Validação de métodos analíticos: estratégia e

discussão. Pesticidas: Revista de

Ecotoxicologia e Meio Ambiente, v. 13, 2003.

https://revistas.ufpr.br/pesticidas/article/vie wFile/3173/2546.

CALDAS, Bárbara Sthéfani et al.

Determinação de açúcares em suco concentrado e néctar de uva: comparativo empregando refratometria, espectrofotometria e cromatografia líquida. Scientia Chromatographica, v. 7, n. 1, p. 53-63, 2015.

http://dx.doi.org/10.4322/sc.2015.016.

CURI, Paula Nogueira et al. Influence of different types of sugars in physalis jellies.

Food Science and Technology, v. 37, n. 3, p. 349-355, 2017.

https://doi.org/10.1590/1678-457x.08816.

DEMIATE, Ivo Mottin et al. Determinação de açúcares redutores e totais em alimentos: comparação entre método colorimétrico e titulométrico. 2002. http://ri.uepg.br:8080/riuepg//handle/12345 6789/574.

DORNEMANN, Guilherme Moraes.

Comparação de métodos para

determinação de açúcares redutores e não-redutores. 2016.

http://hdl.handle.net/10183/143940

FIGUEIREDO, Tânia Margarida Pereira.

Validação de métodos analíticos:

Determinação do teor de açúcar numa amostra de produto alimentar. 2012.

Dissertação de Mestrado. Universidade de

Coimbra, 2012.

http://hdl.handle.net/10316/20637.

GONÇALVES JUNIOR, Vagner.

Desenvolvimento e validação de um método analítico para detecção de carbofurano e 3- hidroxicarbofurano em matrizes biológicas com finalidade forense. 2015. Tese de Doutorado. Universidade de São Paulo, 2015.

https://doi.org/10.11606/D.10.2016.tde01022016-110958.

GONÇALVES, Cristiana et al. Adaptation of dinitrosalicylic acid method to microtiter plates. Analytical Methods, v. 2, n. 12, p. 2046-2048, 2010. https://doi.org/10.1039/C0AY00525H.

GONZÁLEZ, A. Gustavo; HERRADOR, M. Ángeles. A practical guide to analytical method validation, including measurement uncertainty and accuracy profiles. TrAC Trends in Analytical Chemistry, v. 26, n. 3, p. 227-238, 2007. https://doi.org/10.1016/j.trac.2007.01.009. GUIMARÃES, Paulo Ricardo B. Análise de Correlação e medidas de associação. Universidade Federal do Paraná, 2017. https://docs.ufpr.br/ jomarc/correlacao.pdf.

HARRIS, Daniel C. Quantitative chemical analysis. Macmillan, 2010. ISBN: 9781429231237.

IMOTO, Marvina N.; FONTOURA, Paulo SG; FREITAS, RJS. Validação de método multiresíduo para análise de pesticidas organo halogenados em maçãs. Revista do Instituto Adolfo Lutz, v. 63, n. 2, p. 186192, 2004.

http://www.ial.sp.gov.br/resources/insitutoadolfo-

lutz/publicacoes/rial/2000/rial63_2_complet a/995.pdf.

KOKKINIDOU, Smaro et al. The important role of carbohydrates in the flavor, function, and formulation of oral nutritional supplements. Nutrients, v. 10, n. 6, p. 742, 2018. https://doi.org/10.3390/nu10060742.

OLIVEIRA, Yonara Silva Garcia de et al. Comparação entre os métodos laboratorial e portátil na análise da glicemia em felinos com amostras de sangue venoso central e capilar. Ciência Animal Brasileira, v. 16, n. 2, p. 279-286, 2015. 
https://doi.org/10.1590/10896891v16i231266.

SANTOS, Angela Alves dos et al.. Dosagem de açúcares redutores com o reativo DNS em microplaca. Brazilian Journal of Food Technology, 20. 2017. https://doi.org/10.1590/1981-67.

SENGAR, Garima; SHARMA, Harish Kumar. Food caramels: a review. Journal of food science and technology, v. 51, n. 9, p. 1686-1696, 2014. https://doi.org/10.1007/s13197-012-0633-z.

SILVA, Roberto do Nascimento et al. Comparação de métodos para a determinação de açúcares redutores e totais em mel. Food Science and Technology, v. 23, n. 3, p. 337-341, 2003. https://doi.org/10.1590/S010120612003000300007.

SOUSA, Yone Alves et al. Avaliação físicoquímica e microbiológica de polpas de frutas congeladas comercializadas em Santarém-PA. Brazilian Journal of Food Technology, v. 23, 2020.

https://doi.org/10.1590/1981-6723.08518.
Team, R. C. (2013). R: A language and environment for statistical computing. https://repo.bppt.go.id/cran/web/packages/d plR/vignettes/intro-dplR.pdf.

VASCONCELOS, N. M.; PINTO, G. A. S.; DE ARAGAO, F. A. S. Determinação de açucares redutores pelo ácido 3, 5dinitrosalicílico: histórico do desenvolvimento do método e estabelecimento de um protocolo para o laboratório de bioprocessos. Embrapa Agroindústria Tropical-Boletim de Pesquisa e Desenvolvimento (INFOTECAE), 2013.

https://www.infoteca.cnptia.embrapa.br/bits tream/doc/982130/1/BPD13017.pdf.

ZHANG, Qi et al. Direct Carbohydrate Analysis in Beverages and Foods Using Pulsed Amperometric Detection or Charged Aerosol Detection. Thermo Fisher Scientific Publication, v. 7, 2015. http://www.unitylabservices.eu/content/dam /tfs/ATG/CMD/cmd-documents/scires/posters/chrom/lc/sys/PN-71524-LCCarbohydrate-PAD-CAD-Pittcon2015PN71524-EN.pdf. 